\title{
e-Migrinter
}

$14 \mid 2016$

L'ajustement méthodologique comme fabrique critique du savoir dans les études migratoires

\section{Les sources historiques du quotidien en exil}

Vers une relecture de la Grande Émigration polonaise de 1831 en France

\section{Valentin Guillaume}

\section{OpenEdition \\ Journals}

Édition électronique

URL : https://journals.openedition.org/e-migrinter/699

DOI : 10.4000/e-migrinter.699

ISSN : 1961-9685

Éditeur

UMR 7301 - Migrinter

Référence électronique

Valentin Guillaume, «Les sources historiques du quotidien en exil », e-Migrinter [En ligne], 14 | 2016,

mis en ligne le, consulté le 20 mai 2021. URL : http://journals.openedition.org/e-migrinter/699; DOI https://doi.org/10.4000/e-migrinter.699

Ce document a été généré automatiquement le 20 mai 2021.

Tous droits réservés 


\title{
Les sources historiques du quotidien en exil
}

Vers une relecture de la Grande Émigration polonaise de 1831 en France

\author{
Valentin Guillaume
}

\section{NOTE DE L'AUTEUR}

Cet article s'inscrit en parallèle de l'écriture d'une thèse en Histoire, sous la direction de Nancy L. Green à l'EHESS (Paris), qui porte sur les « petits » exilés de la Grande Émigration polonaise de 1831 dans l'Ouest de la France et qui tente d'identifier leurs différentes stratégies d'insertion et les trajectoires migratoires dans la France du XIXe siècle.

Le 29 novembre 1830, de jeunes officiers polonais portés par le vent révolutionnaire qui soufflait sur l'Europe prirent les armes dans les rues de Varsovie. Ce fut l'étincelle qui déclencha l'une des plus importantes luttes de la Pologne pour son indépendance face à l'Empire russe. Après dix mois de conflit et une défaite, ce sont près de 6000 Polonais qui prirent la route de l'exil, majoritairement vers la France (Bielecki, 1986, p. 21). À partir de 1831, s'initie un vaste mouvement migratoire que les historiens polonais appelleront un siècle plus tard, la Grande Émigration (Goddeeris, 2013, p. 13). «Grande », parce qu'en trouvant refuge en France, l'élite de cet exil fit de Paris la seconde capitale polonaise, l'âme "véritable » d'une nation sous domination politique et culturelle (Ponty, 2011, p. 30). Ainsi, Adam Mickiewicz y écrivait ses poèmes, Frédéric Chopin y composait ses nocturnes et le prince Czartoryski tentait d'y exister politiquement. Les membres les plus éminents de cette Grande Émigration voulaient montrer à leurs contemporains que si la Russie avait réprimé l'insurrection et gagné la guerre contre l'indépendance polonaise, elle n'avait pas tué la Pologne. Tel était le message qui alimentait l'ébullition intellectuelle polonaise au XIXe siècle en France; tel était le message derrière le terme de "Wielka Emigracja ", choisi et resté dans la postérité par l'historiographie polonaise pour qualifier et caractériser ce phénomène 
migratoire. La Grande Émigration de 1831 possédait en son sein les plus grandes figures polonaises de l'époque, qui en sont devenues ses représentants. Symboles de la résistance politique et culturelle polonaise, symboles régulièrement réactivés des liens d'amitiés avec une France terre d'asile et accueillante, ces grandes figures polonaises laissèrent peu de place à leurs 6000 compagnons d'exil dans l'historiographie européenne. De même, les sources historiques propres à ces "petits" exilés sont, à l'image de ceux dont elles témoignent, tombées dans l'oubli, reléguées derrière les récits issus d'une élite prestigieuse, sur lesquels s'est appuyée la mémoire collective polonaise. Cette majorité silencieuse et méconnue mérite qu'un regard neuf sur des milliers de documents, pourtant conservés dans les archives françaises, vienne lui redonner toute sa place et finalement offrir à l'exil du XIXe siècle une lecture plus sociale et attentive aux choix et aux trajectoires de l'ensemble de ses représentants.

Dans un premier temps, nous allons expliquer quelles sont ces sources qualifiées de «nouvelles» parce qu'elles demeuraient jusqu'alors inexploitées pour différentes raisons : leurs relations indirectes avec le sujet, leur éparpillement sur le territoire français et le faible intérêt des historiens pour ces exilés invisibles, incapables de s'exprimer politiquement et de faire vivre la nation polonaise en exil à l'image de leurs illustres compatriotes comme l'a retenu l'historiographie européenne. Dans un second temps, nous démontrerons en quoi ces sources apportent de nouvelles clefs de compréhension sur la Grande Émigration polonaise en particulier et sur la migration politique en général. À mesure que l'espoir d'un retour victorieux s'évanouit, nous verrons que l'analyse du quotidien des réfugiés du XIXe siècle dans les villages de l'Ouest de la France permet d'élargir les considérations actuelles sur la migration politique.

\section{D'autres sources historiques pour un autre exil}

Cette majorité silencieuse n'a pas écrit de grands poèmes restés dans les mémoires. Ces " petits » exilés n'ont pas fait le récit de leur lutte « héroïque » pour l'indépendance de leur pays, mais ils ont laissé des traces de leur vie quotidienne au cœur de la société française. Ils écrivaient très souvent. Leur but n'était pas de valoriser leur nation bafouée mais, à la différence de leurs glorieux compatriotes, ils prenaient la plume en permanence pour se justifier. Ce temps de "la preuve et l'épreuve ", décrit par Sylvie Aprile (2010, p. 94), obligea tous les réfugiés polonais à justifier de leur participation à l'insurrection de Novembre et de leur réelle incapacité à retourner dans leur pays, afin d'être autorisés à entrer en France. Une fois la frontière franchie, il leur fallait encore justifier leur grade, leur position sociale passée ou leur situation familiale pour obtenir des aides du gouvernement français (Mondonico, 1995). Autant d'obligations qui ont parfois choqué des Polonais plutôt habitués à des démonstrations de soutien de la part de la population française. Cette dernière soutenait la cause de la Pologne face à son « oppresseur » russe et les souvenirs encore bien présents des épopées communes dans les armées Napoléoniennes étaient propices à l'accueil de ces réfugiés. Pour autant, si tous ces renseignements et cette surveillance étaient peu appréciés des Polonais, cela nous permet aujourd'hui de connaître le profil de ces réfugiés politiques du XIXe siècle. 


\section{Connaître et reconnaître grâce aux sources administratives}

Ces Polonais étaient très souvent des officiers ou d'anciens étudiants, ils avaient majoritairement moins de 30 ans, étaient rarement mariés et venaient tout autant du Royaume de Pologne que de l'ancien Grand-Duché de Lituanie. La défaite des hommes entraîne également des femmes et des enfants à passer la frontière et à quitter leur pays. On observe difficilement leur discrète présence dans les pas de leurs proches. Néanmoins, leur rôle dans le quotidien de leurs maris ou de leurs pères, réfugiés au sein de la société française, ne saurait être négligé. Si nous avons pu préciser les profils sociaux de ces Polonais, c'est parce que le gouvernement français décida de les éparpiller sur une partie du territoire à partir de 1833. En effet, les grands "dépôts " Besançon, Avignon ou Bourges, qui avaient « accueilli » les réfugiés polonais dès 1831, avaient montré leurs limites. Les troubles, provoqués par le rassemblement d'un important nombre de Polonais ${ }^{1}$ en quelques points, avaient décidé le gouvernement de Juillet à les disperser en petits groupes, sur des départements français éloignés des frontières, notamment dans le Centre et l'Ouest de la France. Cette séparation forcée a placé plusieurs milliers de Polonais sous la responsabilité des préfets et des maires de province. Les archives départementales nous ont donc offert un premier contact avec ces réfugiés. Chaque préfecture a conservé, selon le zèle des administrateurs locaux, des dossiers propres à tous les réfugiés politiques dont elle avait eu la charge ${ }^{2}$. Dans les séries « $\mathrm{M}$ » (Fonds de la préfecture; Police des étrangers), se trouvent des listes de réfugiés "accueillis", mais aussi toute une série d'informations sur leurs profils et leurs quotidiens. Il faut dire que dès leur entrée en France, l'administration française comptait et identifiait les réfugiés présents sur son sol. Cela était d'autant plus nécessaire que le gouvernement français de la Monarchie de Juillet avait choisi de distribuer à tous les réfugiés politiques qui justifient de dangers réels à retourner dans leur foyer au regard des représailles russes, des subsides en fonction de leur grade dans l'armée "insurrectionnelle », ou de leur position sociale passée. Ces aides, basées sur les normes de la bienfaisance chrétienne en vertu desquelles, comme le souligne Gérard Noiriel (2006, p. 47), les individus ont des besoins différents selon leur milieu social, nous permettent aujourd'hui de préciser le profil des réfugiés politiques polonais du XIXe siècle.

De plus, ces listes étaient accompagnées de milliers de lettres échangées entre les Polonais, l'administration locale et le ministère de l'Intérieur. En effet, pour connaître l'impact de l'arrivée de ces réfugiés sur la population locale, mais aussi pour inciter les préfets à réduire peu à peu les aides octroyées, le gouvernement réclamait régulièrement des enquêtes sur les conditions de vie des Polonais. C'était également auprès du préfet que les réfugiés polonais devaient s'adresser pour obtenir une autorisation de déplacement. Assignés à résidence dans certaines villes par l'administration départementale, les Polonais ne pouvaient se déplacer que dans le cas où ils disposaient d'un passeport «intérieur ». Cela nous permet aujourd'hui de mieux comprendre les logiques et les raisons de leurs déplacements. Nous nous sommes très vite aperçus que les deux motifs invoqués par les réfugiés polonais du XIXe siècle pour justifier un changement de résidence étaient la santé et le travail. Déposées avec un certificat médical, les demandes de déplacement pour des raisons de santé nous ont permis de souligner que les médecins diagnostiquaient très souvent des problèmes liés aux blessures subies au combat, ainsi que des soucis inhérents à la tristesse, à la mélancolie et à la nostalgie. D'ailleurs, il n'est pas rare de lire qu'un réfugié s'est suicidé 
car il ne supportait plus sa situation d'exilé, ou qu'un autre vit dans la misère, ses blessures l'empêchant de travailler. Au-delà du motif de santé, c'est pour répondre à une opportunité d'emploi que les Polonais multipliaient les demandes de déplacement. La préfecture réclamait alors une attestation signée de l'employeur ou, au moins, un justificatif pour valider le changement de résidence des réfugiés. Cela nous permet aujourd'hui de mieux cibler la période à laquelle les Polonais ont accéléré leur recherche d'emploi et surtout d'identifier les différents secteurs professionnels dans lesquels ils se sont investis.

\section{Une insertion économique appréhendée dans les archives françaises}

Nous avons également pu voir que le gouvernement français encourageait et favorisait l'insertion économique de ces réfugiés politiques. Au-delà des secours réguliers, l'administration offrait ainsi aux Polonais "dignes d'intérêt » par leur modération politique et leur comportement, des aides supplémentaires pour s'inscrire à l'université, pour imprimer une thèse, s'acheter des livres nécessaires à leurs études, ou encore, pour entrer en apprentissage auprès d'un artisan. Les demandes écrites des réfugiés nous permettent de souligner l'importance de ces " coups de pouce » dans les trajectoires professionnelles des Polonais et l'intérêt que l'administration française en retirait. D'après les écrits des préfets du XIXe siècle, cette insertion économique avait le double avantage de réduire considérablement les frais occasionnés par ces Polonais dans le besoin, mais aussi d'éviter une forme d'oisiveté, perçue comme susceptible de nourrir des engagements politiques ou de provoquer des troubles à l'ordre public.

7 Par ailleurs, nous avons choisi de ne pas nous limiter à ces sources administratives. Le manque de moyen des autorités locales, dans la France du XIXe siècle, nous a obligé à interroger d'autres sources toujours plus «indirectes». En effet, il suffisait pour ces Polonais de ne plus toucher les aides de l'État pour ne plus être placé sous la surveillance des autorités et de fait, de disparaître des dossiers administratifs. De même, à partir de 1839, la Monarchie de Juillet donna plus de largesse à la mobilité des réfugiés politiques. Pour faciliter leur insertion économique, freinée par toutes ces démarches administratives, le gouvernement de Juillet fit voter une loi permettant aux réfugiés politiques subventionnés et présents depuis plus de cinq ans en France de se déplacer sans justification. Une évolution législative qui devait nécessairement réduire les interactions entre les Polonais et l'administration française et donc limiter la visibilité de l'historien sur cette population. Ensuite, ces sources peuvent involontairement donner une vision déformée de la réalité. Les autorités locales étaient essentiellement chargées de résoudre les problèmes liés aux réfugiés, de faire remonter à leurs supérieurs ce qui n'allait pas dans leur vie ou encore d'identifier ceux qui pesaient trop lourdement sur le budget selon le gouvernement français. De fait, de nombreux Polonais relativement bien insérés économiquement entrèrent très peu en contact avec l'administration locale chargée des réfugiés et échappent ainsi au regard de l'historien.

8 Ces quelques indices nous ont permis de voir que les Polonais se sont majoritairement imposés dans quatre secteurs professionnels: l'artisanat, l'instruction publique, la médecine et l'administration des ponts et chaussées. Si pour les multiples réfugiés polonais devenus menuisiers, tailleurs ou encore cordonniers, il est difficile 
d'appréhender complètement leurs trajectoires professionnelles, ce n'est pas le cas des autres secteurs professionnels. Grâce aux dossiers des étudiants conservés par les universités de médecine ${ }^{3}$, aux rapports sur les enseignants du ministère de l'Instruction publique $^{4}$, et aux nombreux documents propres aux mutations, aux nominations et aux comportements des fonctionnaires du ministère des Travaux publics ${ }^{5}$, il nous a été possible de mieux comprendre leurs parcours. Les Archives nationales conservent les dossiers individuels de chaque personne ayant travaillé pour l'administration française. Ainsi, il est possible d'identifier leurs motivations, leurs situations professionnelles mais aussi leur position familiale au moment de leur entrée dans la fonction publique, jusqu'à leur retraite ou à l'occasion de rares cas de démission.

\section{Quelles sources historiques, pour quelle insertion sociale?}

9 Il nous a semblé nécessaire de dépasser une nouvelle fois les seules sources administratives pour comprendre l'insertion sociale de ces réfugiés politiques. En effet, même si le statut marital des Polonais était noté dans les dossiers tenus par l'administration française, seules les relations politiques qu'ils nouaient avec la population française étaient véritablement surveillées et donc conservées dans les archives. Pour obtenir des informations complémentaires, nous avons eu recours à l'état civil de chaque ville où ces Polonais se sont mariés, ont eu des enfants, et où ils sont décédés. Tout d'abord, grâce aux tables décennales, nous avons pu souligner l'importance des mariages entre des réfugiés polonais et des Françaises. Ceux-ci concernaient deux tiers des Polonais arrivés en France au début des années 1830. D'ailleurs, il est possible d'observer que les premiers mariages ont été très rapidement célébrés, parfois quelques mois seulement après les premiers pas de ces réfugiés sur le sol français. La très faible présence féminine parmi les réfugiés polonais ainsi que l'image positive de ces «combattants du Nord" auprès de la population française, semblent avoir engendré davantage de mariages mixtes qu'au sein des réfugiés italiens, portugais ou espagnols présents en France au XIXe siècle. Nous avons ensuite choisi de nous intéresser aux actes de mariages et notamment aux témoins mobilisés par les époux. Qu'il s'agisse de voisins, de collègues de travail ou de compatriotes, les signatures en bas de ces actes nous ont permis de connaître un peu mieux les relations sociales que pouvaient entretenir ces réfugiés polonais au quotidien.

L'analyse des états civils a également permis d'identifier les enfants nés de ces mariages. Les fils ou filles de réfugiés polonais ont dû évoluer à leur tour dans la société française et y ont laissé des traces. À Paris par exemple, une école polonaise fut fondée dans les années 1840 pour ces enfants. Les listes des élèves qui fréquentèrent cette école sont encore conservées à la Bibliothèque polonaise de Paris. Dès lors, nous avons pu les comparer aux enfants nés de ces mariages mixtes, en province, afin d'identifier les familles qui pouvaient ou voulaient envoyer leurs fils et filles dans la seule école polonaise en France de l'époque.

11 De même, ces actes de mariage nous montrent dans quelle mesure cette population était coupée de son pays d'origine. À chaque fois que la mairie réclamait un acte de naissance ou les consentements des familles polonaises restées au pays, les contractants étaient obligés d'admettre qu'ils n'avaient plus aucunes nouvelles de leurs proches, que la Russie empêchait toute communication et qu'il leur était même impossible de savoir si leurs parents étaient encore en vie ou non. Ce sont donc des 
compatriotes qui déclaraient, sur l'honneur, que le futur marié était bien célibataire, né à telle date et dans telle ville en Pologne. Le même procédé fut d'ailleurs utilisé par l'administration locale pour connaître la situation des réfugiés polonais au quotidien. Face à leurs faibles moyens, les autorités locales étaient obligées de se renseigner auprès des compatriotes pour avoir des informations sur l'un des leurs. Témoins du manque d'efficacité de l'administration au XIXe siècle, ces déclarations illustrent également les tensions qui ont pu apparaître entre les Polonais eux-mêmes. Que cela soit pour des questions de comportement ou pour des divergences politiques, les dénonciations allaient bon train. La dépendance des réfugiés aux aides du gouvernement, qui pouvaient être supprimées à tout moment, et l'importance d'une bonne « réputation » pour trouver un employeur, poussaient quotidiennement ces personnes à soigner leur image, quitte parfois à se désolidariser de certains de leurs compagnons d'exil. Dès lors, des exilés étaient à l'affût du moindre écart de l'un de leurs compatriotes et les réseaux de solidarité se mettaient alors en marche pour exclure l'un des leurs.

\section{Un éloignement du combat politique à interroger}

12 Il apparaît de manière évidente qu'entre la surveillance de l'administration française d'une part, la censure qui entourait toute publication idéologique d'autre part, ou encore la dispersion des Polonais sur l'ensemble du territoire, il est rapidement devenu difficile de maintenir une politisation au sein de ces réfugiés. Les nombreuses déclarations sur la politique à mener pour « reconquérir » la Pologne, retrouvées à la Bibliothèque polonaise de Paris, ont bien sûr participé à une certaine émulation idéologique au sein de l'élite. Mais les « petits » réfugiés y ont essentiellement participé de manière passive ou indirectement à travers quelques banquets ou enterrements, qui étaient au XIXe siècle des lieux et des temps d'expression politique incontournables (Diaz, 2014, p. 189). Leurs signatures au bas des documents publiés par le courant démocratique, comme par le parti aristocratique du prince Czartoryski, apparaissent finalement comme leur seul et unique investissement politique durant leurs années d'exil. Ces documents témoignent d'une extrême division au sein de l'élite polonaise qui peinait à s'unir autour d'objectifs communs et à rassembler l'ensemble des «petits » exilés. Par ailleurs, en étudiant les quelques duels qui avaient pu avoir lieu entre ces Polonais, nous avons noté qu'une grande partie de ces conflits mortels avait pour origine des divergences politiques.

13 C'est enfin les naturalisations d'une partie de ces réfugiés politiques que nous avons décidé d'interroger. Si les démarches dans ce but étaient rarissimes chez les exilés du XIXe siècle toujours dans l'attente d'un retour glorieux, les réfugiés politiques polonais furent nombreux à franchir le pas et à soumettre des demandes d'admission à domicile ou de naturalisation. Ces demandes ont été très peu évoquées par les récits polonais et sont absentes des archives départementales; ce sont donc les Archives nationales qui nous ont permis de les appréhender au mieux ${ }^{6}$. Les dossiers de naturalisation livrent aussi de nouvelles informations sur la position sociale et l'intégration des personnes qui en ont fait la demande. Par ailleurs, les dates d'ouverture de dossier doivent être analysées au regard de la situation personnelle du réfugié, mais également de l'évolution politique de son pays d'origine. Si depuis 1831 quelques amnisties et autorisations de retour en Pologne pouvaient être accordées par l'Empire Russe, c'est en 1856 que le Tsar décida de les généraliser. Rarement mis en avant par les réfugiés 
eux-mêmes, ces retours «tête basse » et «non victorieux » sont un phénomène qui ne doit pas être négligé. Pour les prendre en compte, il faut revenir à ces nombreux documents administratifs conservés dans les archives départementales, car les Polonais devaient obtenir auprès de l'administration locale un certificat validant leur bonne conduite politique pour que l'ambassade russe à Paris donne son accord. La France payait généralement les lourds frais de voyage qu'occasionnait ce retour au pays, si une demande était réalisée et justifiée. Ces multiples échanges entre les Polonais et l'administration locale permettent de comprendre les choix qui se sont opérés à ces moments précis, mais aussi de mieux connaître ceux qui ont décidé de repartir quand la majorité de leurs compatriotes est restée en France.

Finalement, peu importe qu'il ne s'agisse que d'une lettre pour réclamer une aide financière ou demander une autorisation de déplacement envoyée à un préfet anonyme de la Monarchie de Juillet, d'une signature au bas d'un acte de mariage avec une Française ou d'une rédaction pour un concours de l'administration publique, ces «traces» de vie quotidienne sont autant de témoignages qui offrent l'opportunité de redécouvrir les exilés du XIXe siècle en France. Nous avons choisi de mettre en valeur et de recouper ces témoignages pour comprendre les multiples comportements et les différentes possibilités offertes à ces Polonais au sein de la diplomatie européenne du XIXe siècle, du cadre législatif propre aux réfugiés mis en place à partir de 1832 en France et des différents contextes politiques et économiques locaux.

\section{Des exilés politiques aux immigrés ordinaires}

$15 \mathrm{Au}$ regard des sources utilisées pour appréhender le quotidien de ces réfugiés, ces derniers semblaient bien loin des salons où l'élite polonaise se réunissait à Paris. La capitale française était d'ailleurs interdite aux réfugiés politiques au XIXe siècle, sauf autorisations exceptionnelles. De même, les Polonais étaient rarement plus d'une quinzaine à être assignés à résidence dans une même ville; leur nombre dépendait surtout des capacités de surveillance, du dévouement et du zèle des autorités locales. Nous avons également pu noter que l'administration française n'hésitait pas à isoler les éléments les plus "turbulents» dans des villages de quelques centaines d'habitants. Alors qu'ils avaient franchi les frontières par centaines, les Polonais se sont retrouvés, avec quelques compatriotes et dans des villes qu'ils n'avaient pas choisies, à devoir surmonter les souffrances physiques et morales qui accompagnaient leur défaite puis leur exil. Ils ont ainsi dû très vite s'adapter au marché du travail français pour compenser les faibles secours octroyés par le gouvernement. Alors, au fur et à mesure que l'espoir d'un retour diminua, la position de l'exilé se rapprocha de celle de l'immigré ordinaire (Temine, 1991, p. 57). Ces Polonais n'étaient plus seulement des combattants glorieux et accueillis comme tels voire des réfugiés politiques sous surveillance, ils étaient également devenus des employés à la recherche d'un travail, d'une occupation lucrative. Comme le soulignait Thadée Piotrowski (1947, p. 75), la recherche d'une activité, d'un salaire et d'une formation étaient au cœur de leurs déplacements sur le territoire français. Il ne s'agissait pas de se réunir pour organiser un retour victorieux en Pologne ni de faire vivre un sentiment national, mais de se rendre dans les régions les plus susceptibles d'offrir des emplois. 


\section{Les réfugiés polonais face au marché de l'emploi français}

16 Nos sources nous offrent une vision plus approfondie du rapport qu'entretenait un exilé au XIXe siècle avec le monde du travail du pays d'accueil (Aprile, 2010, p. 179). Les subsides du gouvernement français étant rarement suffisants et en baisse régulière, les réfugiés polonais furent contraints de travailler. Un phénomène d'autant plus intéressant que les réfugiés de cette période n'avaient pas de contrat de travail, ni de spécialisation cohérente avec le marché de l'emploi au sein duquel ils se voyaient contraints de s'insérer. Ils étaient obligés d'évoluer dans des milieux professionnels qui n'étaient pas les leurs avant l'exil, de survivre par le travail quotidien, quitte à délaisser leurs premières considérations politiques et à sacrifier leurs rangs professionnels passés. En effet, si ces « petits » exilés n'évoluaient pas dans le « beau monde » parisien, ils étaient tout de même des petits aristocrates ou des petits bourgeois de la classe sociale supérieure en Pologne. Leur adaptation et leur reclassement sociaux témoignent encore une fois de la particularité de cet «autre exil». Un Polonais de la Grande Émigration pouvait donc être un ouvrier au sein des grands travaux publics de l'époque, ou un médecin formé en France et uniquement préoccupé par la concurrence qui sévit auprès des patients d'un petit village de Bretagne. Il pouvait également faire carrière dans l'administration publique française, à la préfecture ou dans les ponts et chaussées, s'intégrer dans les universités de la métropole, mais aussi être dans l'incapacité de travailler pour cause de blessure ${ }^{7}$. Si l'insertion économique des réfugiés dans le pays d'accueil fut analysée par Émile Temine pour le XXe siècle (Temine, 1991, p. 57), si Sylvie April l'a évoqué à l'endroit de certains exilés français (Aprile, 2010, p. 207), les sources que nous utilisons permettent d'associer également ce phénomène à l'exil du XIXe siècle en France, et ce, avec une ampleur encore peu appréhendée. De plus, l'exilé polonais du XIXe siècle apparait tout à la fois acteur, par ses connaissances et ses préoccupations professionnelles, que dépendant des opportunités que le contexte économique lui offrait. La multiplication générale des effectifs dans les universités de médecine, les débuts de l'apprentissage des langues étrangères que pouvaient enseigner les Polonais aux élèves français dans les collèges royaux, ainsi que la politique de grands travaux publics du XIXe siècle en France, sont autant d'éléments qui témoignent, chez ces réfugiés polonais, d'une certaine capacité à valoriser leurs acquis personnels précédant leur exil en fonction des possibilités offertes par le marché de travail local en France.

Par ailleurs, nous avons souligné que cette insertion sur le marché de l'emploi de la société française avait été souhaitée et provoquée par les différents gouvernements français du XIXe siècle. Cela n'est pas négligeable car ce positionnement politique a permis à de nombreux exilés de progresser dans leurs carrières professionnelles jusqu'à des postes à hautes responsabilités, notamment dans l'administration des ponts et chaussées (Harismendy, 2002, p. 57). Cette dernière a conservé les dossiers des Polonais qui passèrent des examens, furent mutés afin de monter en grade dans différents travaux publics en France $^{8}$, et qui se sont complètement investis dans ce nouvel horizon professionnel. Ces dossiers permettent de mieux comprendre les raisons de naturalisations parmi cette population puisqu'un étranger ne pouvait devenir conducteur embrigadé (permanent) ni toucher une retraite à la fin de sa carrière sans avoir été naturalisé. Ils révèlent encore que certains d'entre eux n'ont pas hésité à retourner temporairement en Pologne ou en Russie pour y travailler au service de 
compagnies de chemin de fer ; des exemples éloignés de l'idée d'un retour victorieux dans un pays libéré.

Enfin, nous devons ajouter que contrairement aux réfugiés espagnols et italiens, l'accueil positif réservé aux Polonais par la population française facilita leur insertion sur le marché de l'emploi. Aussi, la corrélation entre les donateurs français aux souscriptions qui se développèrent à l'égard de la cause polonaise en 1831 et les employeurs qui leur ouvrirent les portes à l'heure de chercher un travail est indéniable. L'expérience militaire, les connaissances mathématiques et linguistiques, ainsi que les études réalisées par cette population dans leur pays d'origine jouèrent un rôle majeur dans les emplois qu'ils occupèrent en France, même si ceux-ci correspondirent rarement aux emplois qu'ils auraient pu espérer obtenir en Pologne. Au-delà de la simple solidarité, les réfugiés polonais, qui savaient en grande majorité écrire, compter, diriger des hommes, étaient particulièrement appréciés des employeurs français.

\section{Des réfugiés politiques devenus des chefs de familles françaises}

Grâce à ces témoignages du quotidien, nous pouvons également affirmer que sur l'ensemble des réfugiés, les Polonais qui se marièrent avec des Françaises connurent une stabilité géographique et professionnelle plus importante. Le passage du statut de simple réfugié à celui de chef de famille obligeait généralement les Polonais à revoir leurs priorités. C'est notamment le cas des réfugiés les plus engagés politiquement qui, selon les rapports de l'administration, diminuaient fortement leur investissement lorsqu'ils se devaient de subvenir aux besoins de leurs enfants, de leurs épouses et même parfois de leurs belles-familles. Grâce à une analyse précise des enfants présents à l'école polonaise de garçons de Paris, nous avons pu observer que la majorité des enfants de réfugiés parlait rarement le polonais et que les élèves qui avaient l'opportunité de "renouer » avec leurs origines étaient souvent issus des familles les plus aisées et les plus à même de venir s'installer à Paris ou de payer l'internat. En effet, même si des bourses furent mises en place, il fallait encore s'investir dans de lourdes démarches administratives et se justifier de son bon comportement politique et social auprès du gouvernement pour en bénéficier. L'étude des états civils a aussi permis de mesurer l'importance des mariages mixtes entre Polonais et Françaises. Cela était rarissime pour les réfugiés d'autres nationalités au XIXe siècle, et également presque impossible un siècle plus tard pour les immigrés économiques polonais, d'après Janine Ponty $(1985$, p. 52). De sorte que ces mariages, tout comme les carrières professionnelles réfléchies, témoignent dans une certaine mesure, d'une volonté peu commune au sein des exilés du XIXe siècle (Aprile, 2010, p. 127) : celle d'accepter de penser son avenir, non pas dans le pays natal, retrouvé et libéré, mais bien dans le nouveau pays d'accueil.

Bien que peu nombreuses, les femmes de ces réfugiés étaient aussi des Polonaises qui avaient suivi leur époux sur la route de l'exil. Malgré un départ dans l'ombre, dans les pas de leurs proches, ces «suiveuses» (Green, 2002, p. 105), presque invisibles, n'en sont pas moins devenues actrices, puisqu'elles influencèrent la trajectoire migratoire de leurs familles. Ainsi, il n'était pas rare de lire qu'un préfet se félicitait que les réfugiés, occupés à leur mariage où à leurs enfants, fassent passer leurs nouvelles responsabilités familiales avant toutes revendications politiques «excessives » et comprennent que leurs positions réclamaient désormais de la prudence. Ces femmes 
travaillaient rarement; ou seulement lorsque l'inactivité de leurs maris devait être compensée ou que la maladie ou les blessures emportèrent leurs proches. Presque invisible dans les sources, la vie quotidienne de ces exilées nécessite une lecture plus attentive, entre les lignes, et dépassant souvent l'objet premier d'un acte de surveillance policier ou d'une correspondance administrative. Ces femmes s'exprimaient rarement mais nous pouvons retrouver certaines de leurs correspondances avec l'administration lorsqu'elles se retrouvaient seules et en détresse ${ }^{9}$. Elles semblent finalement être un pilier sur lequel maris, enfants ou familles pouvaient s'appuyer durant l'exil. À la mort de leur époux, il n'était pas rare de les voir prendre seules la route du retour en Pologne et mettre fin à cet « exil » qui n'avait eu finalement de sens qu'à travers leurs proches.

\section{Penser son avenir en France}

21 Contrairement aux considérations actuelles sur l'exil du XIXe siècle et aux récits mémoriels de l'élite polonaise, les regards des "petits » réfugiés ne restèrent donc pas uniquement tournés vers la Pologne. À mesure que l'exil se prolongea, cette majorité silencieuse de la Grande Émigration commença à avoir un rapport au temps et à son exil différent de celui de ses illustres compatriotes. Penser son avenir en France, ce qui pouvait être considéré comme outrageant au regard du combat mené par certains exilés polonais, s'engager dans une voie professionnelle ou familiale et s'y investir au détriment de l'engagement politique, était pourtant très courant chez ces exilés. Pour preuve, le faible nombre de départs et de réactions de la part des exilés polonais lors de la révolte de Cracovie en 1846, du printemps des peuples en 1848, ou encore de l'insurrection de Varsovie en 1863 que l'administration française surveilla de près. De même, les dossiers professionnels conservés par les différents corps administratifs, par les universités ou par certains employeurs sont, à cet égard, très explicatifs. La présence de ces réfugiés en France ne semblait plus temporaire, comme l'imaginait le gouvernement de Louis-Philippe en 1831, mais elle s'éternisa au point de pouvoir la qualifier de "permanente ». D'ailleurs, lorsqu'en mai 1856, Alexandre II, le nouvel Empereur de Russie, considéré comme plus libéral que son père Nicolas Ier, accorda une amnistie à l'égard des Polonais exilés de 1831, très peu en profitèrent. Il faut savoir que depuis 1835, tous les biens de la majorité des Polonais ayant participé à l'insurrection de 1830, avaient été confisqués (Le Comte d'Angeberg, 1862, p. 971). Dès lors, les insertions professionnelles et sociales en France finirent par faire accepter à ces « petits » exilés de la Grande Émigration, le cadre apolitique et définitif de leur exil. La corrélation entre les départs et les aléas de vie en France, que fournissent les dossiers individuels tenus par les préfets ou par les lettres de ces exilés, et expliquant les motifs de leurs départs, semblent démontrer que c'est même parfois l'échec d'une carrière ou d'un mariage en France qui poussa au retour dans le pays d'origine.

\section{Conclusion}

Finalement, pour cette majorité silencieuse, il a surtout fallu trouver sa place dans ce pays qui n'a jamais véritablement été pour eux une terre de revendications politiques mais plutôt une terre de vie et de survie, d'intégration et/ou de misère. S'il est indéniable que Paris a offert un véritable écho à la résistance culturelle et politique de la nation polonaise au XIXe siècle, il était devenu nécessaire pour les chercheurs de 
sortir des murs de la capitale et des salons parisiens. En s'arrêtant à l'étude des élites polonaises de la Grande Émigration, à leurs investissements politiques et à leurs combats idéologiques, les historiens ont trop longtemps passé sous silence la relation de la majorité des réfugiés polonais avec leur exil, un exil autrement moins prestigieux, mais tout aussi intéressant pour la compréhension des phénomènes migratoires dans leur globalité.

\section{BIBLIOGRAPHIE}

Aprile, Sylvie (2010) Le siècle des exilés, Bannis et proscrits de 1789 à la Commune, Paris, Éditions CNRS, $336 \mathrm{p}$.

Bielecki, Robert (1986) Zarys rozproszenia wielkiej emigracji we Francji 1831-1837, Les contours de la dispersion de la Grande Émigration en France, Varsovie, Panstwowe Wydawnictwo Naukowe, 368 p.

Bielecki, Robert (1995) Slownik biograficzny oficerow powstania listopadowego, in Dictionnaire biographique des officiers de l'insurrection de Novembre (3 Tomes), Varsovie, Wydawnictwo Trio, $405 \mathrm{p}$.

Diaz, Delphine (2014) Un asile pour tous les peuples? Exilés et réfugiés étrangers dans la France au cours du premier XIXe siècle, Paris, Armand Colin, 305 p.

Green, Nancy (2002) Repenser les migrations, Paris, PUF, 144 p.

Harismendy, Patrick (2002) Les réfugiés politiques en Bretagne (1830-1848), Annales de Bretagne et de Pays de l'Ouest, $\mathrm{n}^{\circ}$ 4, pp. 41-58.

Le Comte d'Angeberg (1862) Recueil des traités, conventions et actes diplomatiques concernant la Pologne 1762-1862, Paris, Amyot, $1171 \mathrm{p}$.

Mondonico, Cécile (1995) L'asile sous la Monarchie de Juillet, Les réfugiés étrangers en France de 1830 à 1848, Paris, EHESS, 477 p., Th. Doct. : Histoire : Paris : 1995.

Noiriel, Gérard (2006) Réfugiés et sans papiers, La République face au droit d'asile, XIXe -XXe siècle, Paris, Hachette, $384 \mathrm{p}$.

Piotrowski, Thadée (1947) Documents pour l'étude de l'immigration polonaise de 1831 en France, in INED, Documents sur l'immigration, Paris, PUF, pp. 43-75.

Ponty, Janine (1985) Une intégration difficile : Les Polonais en France dans le premier XXe siècle, Vingtième siècle, $\mathrm{n}^{\circ} 7$, pp. 51-58.

Ponty, Janine (2011) Polonia, Des Polonais en France de 1830 à nos jours, Paris, CNHI, 194 p.

Temine, Émile (1991) Émigration politique et émigration économique, in Groppo, B. (Dir.)

L'émigration politique en Europe aux XIXe et XXe siècles, Rome, École française de Rome, pp. 57-71. 


\section{NOTES}

1. Au printemps 1833, environ 300 Polonais s'évadèrent du dépôt de Besançon afin de rejoindre l'insurrection qui éclata à Francfort-sur-le-Main (Confédération germanique).

2. Archives départementales, série 4M.

3. Archives départementales, série 15T.

4. Archives nationales, F17, Instruction publiques.

5. Archives nationales, F14, Travaux publics - Dossiers des conducteurs des Ponts-et-Chaussées.

6. Archives nationales, sous-série BB/11.

7. Archives départementales, série $4 \mathrm{M}$.

8. Archives nationales, F14, Travaux publics - Dossiers des conducteurs des Ponts-et-Chaussées.

9. Archives départementales, série 4M.

INDEX

Index géographique : France

Mots-clés : histoire, réfugiés politiques, vague migratoire, sources, méthodologie, Polonais

\section{AUTEUR}

\section{VALENTIN GUILLAUME}

Doctorant en Histoire, EHESS Paris

valentinguillaume9@gmail.com 